\title{
Primary Breast Tuberculosis: Report of a Case
}

\author{
Kamil Gulpinar*, S. Erpulat Ozis, Suleyman Ozdemir, Atilla Korkmaz \\ Department of General Surgery, Ufuk University School of Medicine, Ankara, Turkey \\ Email: *kamilgulpinar@gmail.com
}

Received October 30, 2012; revised December 1, 2012; accepted December 10, 2012

\begin{abstract}
Mammary tuberculosis is a rare clinical entity, often mimicking breast cancer or abscesses. A 26-year-old female presented with a painful left breast mass and an associated sinus tract with purulent discharge. Physical examination revealed a tender, erythematous, firm left breast mass with an associated sinus tract opening with discharge within the upper outer quadrant of her left breast with a clinically palpable left axillary lymphadenopathy. Ultrasound revealed nonspecific findings and mammography could not utilize due her young age. A surgical procedure was planned and the left breast mass was completely removed by an open surgical biopsy including left axillary lymphnodes and the hystopathological evaluation of the specimen revealed findings consistent with mammary tuberculosis whereas no other nidus for tuberculosis were found within the organs including lungs. Primary mammary tuberculosis should be considered in the differential diagnosis of any case of a painful breast mass that appears refractory to conventional therapy.
\end{abstract}

Keywords: Primary Mammary Tuberculosis; Breast

\section{Introduction}

Mammary tuberculosis is a rare clinical entity, often mimicking breast cancer or abscesses of benign or malignant origin. While mammary tuberculosis is globally reported to account for less than $0.1 \%$ of all known breast diseases, it is reported in developing countries to comprise up to $3 \%$ of treatable breast lesions [1-4].

The breast can be the primary site but more commonly secondary that spreads to the breast through the lymphatic system from axillary, mediastinal or cervical lymph nodes, or directly from underlying structures such as the ribs.

In the literature, radiological features of breast tuberculosis were presented in only a few reports [5-10].

The aim of this report was to detail our experience and to emphasize the diagnostic difficulty of a rare case of tuberculosis of the breast.

\section{Case Report}

A 26-year-old female patient presented with a chronic 7 month history of a painful left breast mass, an associated sinus tract with purulent discharge and low-grade fever. She had no family history of breast cancer, was divorced and had no history of pregnancy or breast-feeding. During that same period, she had been evaluated by several other clinicians. A fine needle aspiration biopsy (FNA) were performed during that period but was unsuccessful

\footnotetext{
${ }^{*}$ Corresponding author.
}

as the pathology report resulted in insufficient material. She had also prescribed empiric antibacterial therapy for mastitis but there were no relief of her symptoms during that period. Physical examination revealed a tender, erythematous, firm left breast mass approximately $30 \times 40$ $\mathrm{mm}$ in diameter with an associated sinus tract opening with discharge within the upper outer quadrant of her left breast (Figure 1). She had also clinically palpable left axillary lymphadenopathy.

Routine hematologic and biochemical parameters were normal, including negative testing for HIV. Left breast

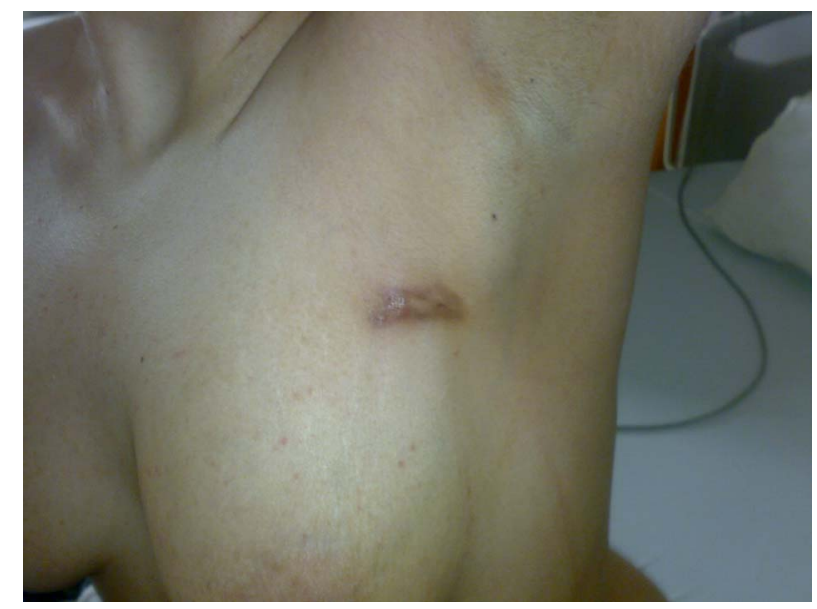

Figure 1. A tender, erythematous, firm left breast mass approximately $30 \times 40 \mathrm{~mm}$ in diameter with an associated sinus tract opening with discharge within the upper outer quadrant of her left breast. 
ultrasound revealed an ill defined, hypoechoic, heterogeneous lesion resembling abscess formation, measuring 42 $\times 14 \mathrm{~mm}$ in size and located in the upper-outerquadrant of the left breast. USG also revealed three enlarged left axillary lymph nodes (measuring $24 \times 21 \mathrm{~mm}$ and $15 \times$ $11 \mathrm{~mm}$ ) with associated microcalcification. A mammography evaluation could not be done as for the patients' request according to her young age. An FNA was utilized and revealed inflammatory cells within purulent material.

Consequently a surgical procedure with an excision of the collection is planned and the left breast mass is completely removed by an open surgical biopsy including left axillary lymphnodes (Figure 2). The hystopathological evaluation of the specimen revealed findings consistent with mammary tuberculosis which is granulomas with central caseaous necrosis, epitheloid histiocytes, Langhans' giant cells, and an intense lymphocytic infiltration at the periphery of the granulomas (Figure 3). Plain-film chest radiography and high resolution computed tomo graphy of the chest were found to be normal.

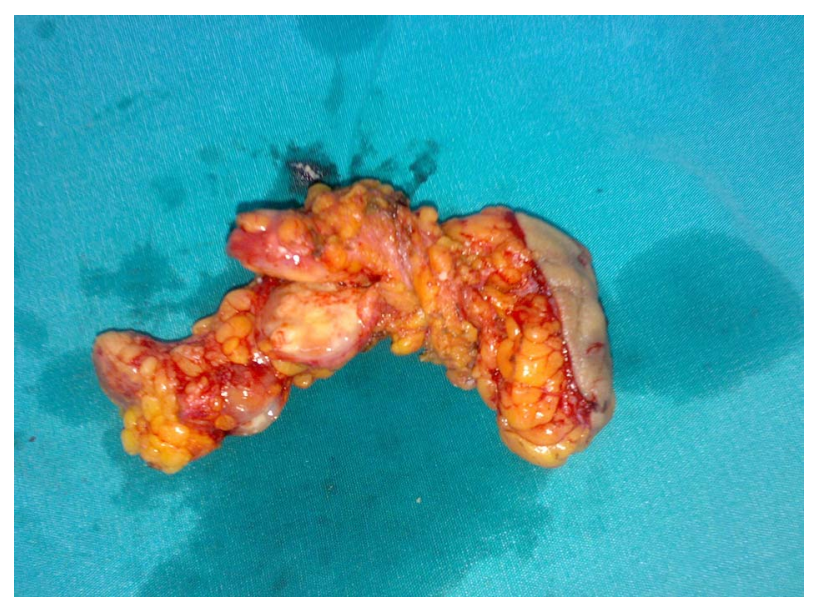

Figure 2. Surgical specimen of the left breast mass completely removed by an open surgical biopsy including left axillary lymphnodes.

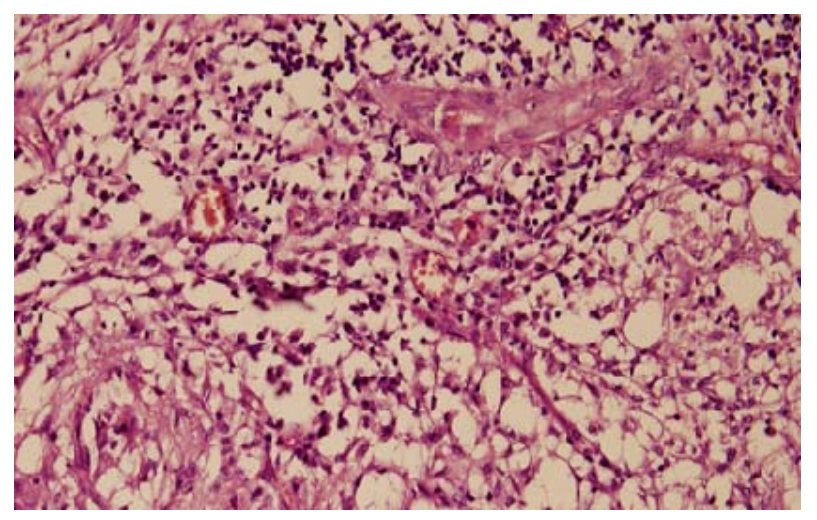

Figure 3. Hystopathological evaluation of the specimen: Granulomas with central caseaous necrosis, epitheloid hystiocytes, Langhans' giant cells, and an intense lymphocytic infiltration at the periphery of the granulomas.
She started antituberculosis drug regimen immediately after hystopathological confirmation, consisting of rifampicin, isoniazid, pyrazinamide, and ethambutol for two months and was followed by rifampicin and isoniazid for an additional four months,. Her physical examination and radiologic evaluation after six months were all found to be normal without any evidence of residual disease including resolution of her left axillary adenopathy.

\section{Discussion}

Breast tuberculosis is a rare clinical entity with incidence ranging from $0.1 \%$ in developed countries, to $0.3 \%-5 \%$ in endemic regions [11]. The disease is more frequently seen in women between 20 and 50 years of age, especially among multiparous and lactating females where the breast is more sensitive to infection and trauma [12].

The clinical signs of mammary tuberculosis can be insidious and nonspecific and often simulate signs of breast carcinoma. Mammary tuberculosis usually affects young, multiparous, lactating women although it may also be seen in males in $4.5 \%$ of cases [13].

Primary infection of the breast may occur through skin abrasions or through the ducts of the nipple [14]. Interestingly, our presented case here is a young nulliparous woman, naturally had no history of lactation that could predispose to mammary tuberculosis. In addition she was diagnosed as primary breast tuberculosis which is extremely rare [5]. Secondary tuberculosis of the breast, on the other hand, is more common and results from hematogenous spreading, retrograde spread from axillary lymph nodes or direct extension from the lung, pleura, mediastinum and articular lesions [9]. Our case did not have previous or active pulmonary tuberculosis and no evidence of the origin of tuberculosis elsewhere were detected.

Clinical presentation of mammary tuberculosis is extremely variable, often presenting as round nodular lumps mainly in the upper outer quadrant of the breast [15].

These lumps are usually covered with indurated tissue, often with fistula formation, but are rarely associated with pain and breast discharge. The borders of the lump are usually irregular while fixation of the lesion to the skin, the underlying muscle or even to the chest wall often poses clinical problems in differentiation from breast carcinoma [16].

In its complex form, breast tuberculosis is characterized by invasion of the skin, with skin and nipple retraction creating the peau d'orange sign resembling breast malignancy.

Based on radiological and clinical characteristics the disease can be divided into three forms: nodular, diffuse and sclerosing [17]. The nodular form is characterized by 
a circumscribed lesion in the breast with an oval tumor shadow on mammography, a finding which can rarely be differentiated from breast cancer. The diffuse form of the disease, also known as disseminated tuberculosis mastitis, is characterized by multiple tuberculous foci of the breast which often cause multiple ulcerations and discharging sinuses on the skin; this form simulates inflammatory breast cancer on mammographic findings. The sclerosing form of the disease is more frequently seen in elderly women and is characterized by an excessive fibrotic process [17].

Mammography and ultrasonography are helpful but unreliable in differentiating mammary tuberculosis from carcinoma [5]. Mammography might be helpful but refused by the patient concern of X-ray expose.

Lesions due to tuberculosis have no specific ultrasonographic findings. In ultrasonography, they are observed as heterogeneous, hypoechoic, irregular bordered masses with internal echoes, or sometimes as thickwalled cystic lesions that show internal septa and posterior acoustic enhancement. In some cases, fistulas and thickening of Cooper ligaments and subcutaneous tissue were reported [5,9].

Furthermore, the relation between the lesion and the chest wall might be evaluated. In follow-up ultrasonography examination of the patient revealed irregular bordered, heterogeneous hypoechoic, focal lesions that were connected to each other and fistula that extended to the skin. Some of the lesions showed posterior acoustic enhancement.

Fine-needle aspiration cytology can be diagnostic for tuberculosis if sufficient material is aspirated. Other types of biopsy, such as core needle or surgical biopsy can get higher accuracy in diagnosis

Fine needle aspiration biopsy or core needle biopsy which could have raised the possible diagnosis of breast tuberculosis were not performed in our patient by our team prior to surgery, due to non-specific inflammatory results and insufficient prior aspiration biopsy in an other center. Surgical resection for diagnosis and treatment was reasonable for our patient as previous such interventions were unsuccessful.

Anti-tuberculosis therapy, consisting of the same regimen used in pulmonary tuberculosis [18] was applied only after the final hystopathological report was received.

\section{Conclusion}

Mammary tuberculosis should be considered in the differential diagnosis of any case of a painful breast mass, mastitis, or breast abscess that appears refractory to conventional therapy. Its recognition and differentiation from breast cancer is particularly important. Physical examination, USG and CT are helpful diagnostic tools on this process. Accurate and final diagnosis should be confirmed by hystopathological examination.

\section{REFERENCES}

[1] R. Khanna, et al., "Mammary Tuberculosis: Report on 52 Cases,” Postgraduate Medical Journal, Vol. 78, No. 921, 2002, pp. 422-424. doi:10.1136/pmj.78.921.422

[2] S. N. Banerjee, et al., "Tuberculous Mastitis: A Continuing Problem,” World Journal of Surgery, Vol. 11, No. 1, 1987, pp. 105-109. doi:10.1007/BF01658471

[3] S. R. Shinde, R. Y. Chandawarkar and S. P. Deshmukh, "Tuberculosis of the Breast Masquerading as Carcinoma: A Study of 100 Patients," World Journal of Surgery, Vol. 19, No. 3, 1995, pp. 379-381. doi:10.1007/BF00299163

[4] S. H. Harris, et al., "Mammary Tuberculosis: Analysis of Thirty-Eight Patients,” ANZ Journal of Surgery, Vol. 76, No. 4, 2006, pp. 234-237. doi:10.1111/j.1445-2197.2006.03692.x

[5] F. Zandrino, F. Monetti and N. Gandolfo, "Primary Tuberculosis of the Breast. A Case Report,” Acta Radiologica, Vol. 41, No. 1, 2000, pp. 61-63. doi:10.1080/028418500127344768

[6] P. Schnarkowski, et al., "Tuberculosis of the Breast: US, Mammographic, and CT Findings,” Journal of Computer Assisted Tomography, Vol. 18, No. 6, 1994, pp. 970-971. doi:10.1097/00004728-199411000-00023

[7] D. J. Crowe, M. A. Helvie and T. E. Wilson, "Breast Infection. Mammographic and Sonographic Findings with Clinical Correlation,” Investigative Radiology, Vol. 30, No. 10, 1995, pp. 582-587. doi:10.1097/00004424-199510000-00003

[8] D. Makanjuola, et al., "Mammographic Features of Breast Tuberculosis: The Skin Bulge and Sinus Tract Sign,” Clinical Radiology, Vol. 51, No. 5, 1996, pp. 354358. doi:10.1016/S0009-9260(96)80115-2

[9] K. K. Oh, J. H. Kim and S. H. Kook, "Imaging of Tuberculous Disease Involving Breast,” European Radiology, Vol. 8, No. 8, 1998, pp. 1475-1480. doi:10.1007/s003300050578

[10] C. Romero, et al., "Mammary Tuberculosis: Percutaneous Treatment of a Mammary Tuberculous Abscess," European Radiology, Vol. 10, No. 3, 2000, pp. 531-533. doi:10.1007/s003300050091

[11] H. F. Hamit and T. H. Ragsdale, "Mammary Tuberculosis," Journal of the Royal Society of Medicine, Vol. 75, No. 10, 1982, pp. 764-765.

[12] I. Maroulis, et al., "Mammary Tuberculosis Mimicking Breast Cancer: A Case Report," Journal of Medical Case Reports, Vol. 2, 2008, p. 34. doi:10.1186/1752-1947-2-34

[13] C. Jaideep, M. Kumar and A. K. Khanna, "Male Breast Tuberculosis,” Postgraduate Medical Journal, Vol. 73, No. 861, 1997, pp. 428-429. doi:10.1136/pgmj.73.861.428

[14] S. P. Luh, et al., "Primary Tuberculous Infection of Breast: Experiences of Surgical Resection for Aged Patients and Review of Literature," Journal of Zhejiang University 
Science B, Vol. 8, No. 8, 2007, pp. 580-583. doi:10.1631/jzus.2007.B0580

[15] H. S. Shukla and S. Kumar, "Benign Breast Disorders in Nonwestern Populations: Part II-Benign Breast Disorders in India,” World Journal of Surgery, Vol. 13, No. 6, 1989, pp. 746-749. doi:10.1007/BF01658426

[16] S. Kervancioglu, et al., "Primary Tuberculosis of the Breast," Diagnostic and Interventional Radiology, Vol.
11, No. 4, 2005, pp. 210-212.

[17] E. Goksoy, et al., "Tuberculosis of the Breast," European Journal of Surgery, Vol. 161, No. 7, 1995, pp. 471-473.

[18] M. Tewari and H. S. Shukla, "Breast Tuberculosis: Diagnosis, Clinical Features \& Management,” Indian Journal of Medical Research, Vol. 122, No. 2, 2005, pp. 103110. 